\title{
Problemi metodologici nello studio del processo psicoterapico e nella valutazione dell'attaccamento e del rischio psicopatologico in adolescenza
}

\author{
Riccardo Williams, ${ }^{1}$ Davide Belluardo, ${ }^{1}$ Fiorella Fantini, ${ }^{1}$ Valentina Postorino, ${ }^{1}$ \\ Francesca Ortu ${ }^{1}$
}

\section{Sommario}

Il gruppo di ricerca che attualmente opera presso il Dipartimento di Psicologia Dinamica e Clinica, ha orientato il proprio lavoro fondamentalmente attorno a due tematiche: lo studio multidimensionale del processo terapeutico (in una prospettiva tanto empirica quanto di ricerca concettuale); la rilevanza della teoria dell'attaccamento e dei sistemi motivazionali per la comprensione dello sviluppo della patologia di personalità e delle sue manifestazioni nel contesto clinico, con particolare riferimento all'adolescenza.

\section{Parole chiave}

Processo, CCRT, adolescenza, teoria dell'attaccamento, sistemi motivazionali

1 "Sapienza" Università di Roma

Corrispondenza: Riccardo Williams

E-mail: riccardo.williams@uniroma1.it 
Il nostro gruppo di ricerca ha orientato il proprio lavoro fondamentalmente attorno a due tematiche: la ricerca in psicoterapia (in una prospettiva tanto empirica quanto di ricerca concettuale) e gli sviluppi della teoria dell'attaccamento, approfondendo in particolare le problematiche legate alla valutazione clinica del rischio psicopatologico in adolescenza. Ne presentiamo una sintetica rassegna.

\section{Ricerca in psicoterapia}

Le ricerche condotte in quest'ambito riguardano aspetti essenzialmente metodologici della valutazione del processo in psicoterapia e hanno utilizzato come strumento privilegiato il CCRT di Luborsky per lo studio del processo psicoterapico e, in particolare, per una valutazione empirica dei principali paradigmi di transfert e delle variazioni di tali paradigmi nel corso di trattamenti psicoterapeutici.

Il metodo del CCRT (Luborsky \& Crits-Christoph, 1990), che mira a inferire i "contenuti mentali inconsci" del paziente analizzandone i significati cognitivi e affettivi cosi come emergono nel corso del lavoro terapeutico, permette di dare una valutazione empirica delle narrative dei pazienti in termini di episodi relazionali, in cui vengono identificati i principali desideri, bisogni e intenzioni del parlante, le risposte dell'altro e le risposte del sé; esso si presenta come uno strumento notevolmente flessibile capace di monitorare l'evoluzione di alcuni processi intrapsichici del paziente (Dazzi, De Coro, Ortu, Andreassi, Cundari, Ostuni, Petruccelli, \& Sergi, 1998b).

Negli studi preliminari, volti a indagare le proprietà psicometriche dello strumento su campioni di soggetti italiani in psicoterapia, abbiamo proposto una modifica del CCRT consistente nell'ampliamento dei criteri di inclusione utilizzati da Luborsky, così da cogliere elementi di rilevanza clinica contenuti nelle comunicazioni del paziente non considerate nel metodo classico. In questi primi lavori, concentrati sulla 
individuazione dell'unità di analisi e presentati ai convegni SPR (Ortu, Cascioli, \& Pazzagli, Piscicelli, Williams, \& Dazzi, 2001; Ortu, Lingiardi, Pazzagli, Williams, \& Dazzi, 2001; Williams, Ortu, Lingiardi, \& Dazzi, 2004) ci siamo interrogati sull'utilità di utilizzare sia narrative relazionali con un indice di completezza basso, inferiore a quello classicamente utilizzato, sia racconti di interazione con partner non umani. Abbiamo inoltre sottolineato la necessità di prestare attenzione alla formulazione linguistica delle categorie e ne abbiamo dato un'esemplificazione clinica. Un successivo lavoro ha studiato la riorganizzazione delle categorie che definiscono la componente Wish del CCRT (W) in funzione della teoria dei sistemi motivazionali di Lichtenberg (1989). Approfondendo un lavoro di De Coro, Andreassi e Dazzi (2001), che introduceva nella W nuove categorie (in particolare relative all'area della sessualità) e proponeva una riformulazione e riorganizzazione delle categorie dei Wish nei termini di sistemi motivazionali che si rivelava capace di discriminare tra le diverse categorie diagnostiche, abbiamo valutato la validità del nuovo sistema applicandolo all'analisi del materiale pubblicato da Lichtenberg in "Lo scambio clinico" (Lichtenberg, Lachmann, \& Fosshage, 1996). Questo lavoro ha fatto emergere un sostanziale accordo fra il quadro motivazionale descritto dallo strumento e la formulazione clinica proposta da Lichtenberg (1989; Lichtenberg, Lachmann, \& Fosshage 1996), accordo che può essere letto come un'indicazione della validità dello strumento proposto.

A partire poi dall'ipotesi che significativi spostamenti nei paradigmi dominanti di transfert siano precondizioni e indicatori di cambiamenti psichici strutturali, abbiamo condotto diversi lavori unendo al CCRT altri strumenti capaci di cogliere diversi fattori importanti per lo studio del processo (per esempio, alleanza terapeutica, stile difensivo). In collaborazione con i gruppi di ricerca di Lingiardi e De Coro abbiamo studiato le caratteristiche delle narrative prodotte dal paziente in 
psicoterapia utilizzando il metodo del CCRT (Luborsky \& Crits-Cristoph, 1990), la Referential Activity (RA) di Wilma Bucci (1997a, 1997b) - che valuta la misura in cui l'esperienza emotiva "è attiva nella mente di chi parla, catturandone le oscillazioni e valutandone l'efficacia nello scambio verbale tra paziente e terapeuta" - e la Defence Mechanism Rating Scale (DMRS) di Christopher Perry (1990; cfr. Lingiardi \& Madeddu, 2002).

Questo tipo di lavoro mirava a un'elaborazione metodologica capace di monitorare lo sviluppo del processo lungo dimensioni differenti: i contenuti tematici delle narrative del paziente e le variazioni nello stile comunicativo come fluttuazioni nelle connessioni referenziali. Per esempio, in un lavoro (Williams, Ortu, Lingiardi, \& Dazzi, 2004), l'applicazione del CCRT e della RA all'analisi dei primi due anni del trattamento di una giovane donna con diagnosi di Disturbo Narcisistico di Personalità, ha mostrato come strumenti molteplici offrano informazioni su aspetti differenti del processo terapeutico e dunque un'analisi maggiormente dettagliata e critica del processo stesso, colgano differenti livelli di funzionamento e prestino attenzione alla relazione complementare tra le dimensioni interpersonale e intrapsichica, in quanto dimensioni fondanti il processo psicoterapeutico stesso.

Successivamente, in collaborazione con il gruppo coordinato da Lingiardi, abbiamo avviato una linea di ricerca sul cambiamento clinico, focalizzandoci sullo studio dell'alleanza terapeutica intesa come dimensione emergente nel campo intersoggettivo che si viene a stabilire tra paziente e terapeuta e che, per questa sua natura, presenta un carattere ciclico in cui si succedono fasi critiche e continue ristrutturazioni (Colli \& Lingiardi, 2001, 2002). Abbiamo così condotto alcuni lavori di ricerca mirati a valutare: 1) l'andamento dell'alleanza rispetto alle organizzazioni difensive e agli schemi relazionali in pazienti appartenenti a diverse categorie diagnostiche (Ortu, Williams, \& Dazzi, 
2004); 2) l'andamento dell'alleanza rispetto al processo nelle due terapie esaminate (Williams, Saracino, Traverso, Ortu, \& Dazzi, 2005). In questi lavori per la valutazione dell'alleanza terapeutica abbiamo utilizzato l'IVAT (Indice di Valutazione dell'Alleanza Terapeutica, oggi CIS Collaborative Interactions Scale; Colli \& Lingiardi, 2009), che permette di identificare, sulla base dei punteggi assegnati (da giudici indipendenti) alle scale dello strumento, gli indicatori - diretti e indiretti - di rottura dell'alleanza terapeutica da parte del paziente e gli interventi di ricomposizione della stessa da parte del terapeuta (Colli \& Lingiardi, 2001, 2002, 2009).

Per quanto riguarda la valutazione multidimensionale del processo (Ortu et al., 2002), abbiamo continuato a riferirci a costrutti elaborati nell'ambito della tradizione di ricerca sulle psicoterapie psicodinamiche: il CCRT di Luborsky, la DMRS di Perry e la RA della Bucci. Gli strumenti citati sono stati applicati per la valutazione di due psicoterapie omogenee rispetto alla seguenti variabili: a) tipologia del trattamento (psicoterapia psicodinamica con frequenza bisettimanale), b) organizzazione di personalità del paziente e del terapeuta, ma diverse rispetto all'esito (drop out vs a termine). Per le siglature sono stati utilizzati trascritti estratti, per entrambe le terapie, da tre fasi successive dei trattamenti. I risultati di questo lavoro (Ortu et al., 2002) ci hanno incoraggiato a concludere che il metodo consente una valutazione della rilevanza delle dimensioni processuali considerate ai fini dello stabilirsi e dell'evolversi dell'alleanza terapeutica nel corso della seduta; inoltre offrivano dati utili per analizzare l'intreccio fra la qualità della relazione intersoggettiva, così come desunta dalla valutazione dell'alleanza terapeutica, e le tre dimensioni del processo, considerate singolarmente e nel loro insieme. In un successivo lavoro ci siamo focalizzati sul ruolo dell'alleanza terapeutica nello sviluppo del processo terapeutico e del cambiamento clinico, con l'obiettivo di identificare $\mathrm{i}$ fattori che influenzano la formazione e l'evoluzione 
dell'alleanza, prestando particolare attenzione alla tecnica adottata dal clinico nelle terapie con pazienti borderline. Abbiamo così applicato a trascritti audioregistrati di sedute, estratti da tre fasi successive di psicoterapie psicodinamiche di due pazienti diagnosticati con la SWAP200 (Westen, Shedler, \& Lingiardi, 2003):

- una scala per la valutazione degli interventi del terapeuta, derivata dalla classificazione proposta da Horowitz (cfr. Williams et al., 2004) a partire dal continuum supportivo-espressivo;

- una scala per riconoscere e quantificare l'alternarsi di rotture e riparazioni dell'alleanza terapeutica (IVAT; Colli \& Lingiardi, 2002).

I risultati ottenuti hanno permesso una valutazione qualitativa della complessa relazione tra alleanza e strategia terapeutica, confermando da un lato la relazione tra interventi supportivi e buona qualità dell'alleanza, in particolare nelle fasi iniziali della terapia, e dall'altra la necessità di riconsiderare la distinzione classica tra interventi supportivi ed espressivi, data la difficoltà di isolare chiaramente i due tipi di interventi.

Più di recente, abbiamo spostato la nostra attenzione sulla valutazione clinica in adolescenza, e sui problemi metodologici da essa sollevati. In questa prospettiva, abbiamo condotto uno studio mirato a confrontare la sensibilità clinica e la validità discriminante di due metodi di valutazione della patologia di personalità in adolescenza, confrontando la procedura standard della SWAP-200 con la valutazione per prototipi, al fine di verificare la validità di costrutto e la validità convergente e discriminante del metodo di valutazione SWAP-200-A per prototipi della patologia di personalità in adolescenza. I risultati ottenuti (Williams, Ferrara, Aloi, Gazzillo, \& 2009) ci hanno permesso di sostenere che la valutazione per prototipi SWAP-200-A conserva lo stesso potere discriminante della procedura standard rispetto ai criteri di validazione ovvero: a) corrispondenza con i criteri dell'Asse II del 
DSM-IV-TR (APA, 2000), b) previsione di comportamenti adattativi e disadattativi nelle diverse aree di funzionamento e nell'adattamento complessivo, c) concordanza con i profili sintomatici dei pazienti. La procedura per prototipi sembra tuttavia essere meno sensibile rispetto alla procedura standard nel cogliere alcune differenze all'interno del cluster B, soprattutto per quanto riguarda la disregolazione emotiva e lo stile antisociale-psicopatico (Williams, Aloi, Di Chio, Ortu, \& Lingiardi, 2008; Williams et al., 2009).

\section{Valutazione clinica in adolescenza alla luce della teoria dell'attaccamento}

La teoria dell'attaccamento ha fornito una cornice di comprensione fondamentale per la valutazione del rischio psicopatologico e per i processi adattativi nelle diverse fasi del ciclo di vita.

Gli studi in questo ambito hanno messo in luce che l'insicurezza dell'attaccamento costituisce un fattore di vulnerabilità per lo sviluppo di diverse condizioni psicopatologiche (Sroufe, 2005). Dopo avere identificato la relazione specifica tra i diversi modelli di attaccamento e le singole condizioni psicopatologiche, lo sforzo degli autori è attualmente rivolto all'impiego delle valutazioni dell'attaccamento nel contesto clinico (Steele \& Steele, 2010).

A tal proposito l'applicazione degli strumenti di valutazione dell'attaccamento al contesto clinico dell'adolescenza ha mostrato diversi tipi di problemi (Barone \& Del Corno, 2007). Tali problemi riguardano prevalentemente la relativa inadeguatezza degli strumenti di valutazione dell'attaccamento nel cogliere alcune caratteristiche essenziali dello sviluppo affettivo e sociale dell'adolescente. In parte, tale difficoltà risulta da un modello delle differenze individuali che è molto proficuo in termini di ricerca empirica, ma non sembra fornire indicazioni sufficientemente specifiche per la comprensione dei processi 
nelle diverse condizioni psicopatologiche e nelle applicazioni ai casi singoli.

Il lavoro di ricerca del gruppo, concentrato negli ultimi anni sulla valutazione clinica in adolescenza, si è sempre rivolto alla teoria dell'attaccamento come cornice teorica di riferimento. Il gruppo ha cioè preso le mosse dallo studio della stabilità ed evoluzione degli schemi cognitivo-affettivi delle relazioni familiari (modelli di attaccamento, secondo la teorizzazione di Bowlby e la metodologia di Ainsworth e Main). In questo senso, come testimoniato da diverse comunicazioni a convegni e pubblicazioni, il lavoro di ricerca condotto in collaborazione con Dazzi e De Coro è consistito in uno studio analitico delle caratteristiche formali - con specifico riferimento alla dimensione della Coerenza del Trascritto - dell'Adult Attachment Interview di Main e Goldwin (1998). Questo sforzo di ricerca ha portato a identificare due fattori principali ("informazione confusa e ridondante", Incoerenza di tipo E, e "informazione incompleta e contraddittoria", Incoerenza di tipo D) responsabili delle specifiche violazioni di coerenza capaci di discriminare le interviste dei soggetti sicuri da quelle degli insicuri (De Coro, Ortu, Speranza, Andreassi, \& Pazzagli, 2003).

Utilizzando anche in quest'ambito una strategia di ricerca rivelatasi fruttuosa rispetto alla valutazione del processo in psicoterapia, abbiamo approfondito lo studio delle caratteristiche formali dell'Intervista sull'Attaccamento mediante il metodo dell'Attività Referenziale di Wilma Bucci (1997a, 1997b), la Defense Mechanisms Rating Scale di Christopher Perry (1990; cfr., Lingiardi \& Madeddu, 2002) e la Scala della Funzione Riflessiva di Fonagy, Steele, Steele, \& Target (1998). Questa strategia di analisi ci ha permesso di identificare differenti modalità di esclusione difensiva, caratteristiche dei diversi stati della mente rispetto all'attaccamento. Questa impostazione generale consente di valutare con maggiore accuratezza la rilevanza che i processi dell'attaccamento hanno per lo svolgimento del processo terapeutico, 
così come studiato alla luce dei costrutti più tipicamente impiegati nello studio sui micro-processi.

Abbiamo d'altra parte approfondito le implicazioni cliniche della teoria dell'attaccamento mettendo a punto un metodo di valutazione del processo psicoterapeutico basato sull'individuazione, nelle trascrizioni parola per parola di alcune sedute psicoterapeutiche, degli indici della coerenza conversazionale utilizzati nella valutazione della A.A.I. di Main e Goldwin (1998).

Un ulteriore approfondimento di ricerca portato avanti in collaborazione con Anna Maria Speranza ha riguardato lo studio della validità convergente fra l'intervista sull'attaccamento (AAI) e alcuni strumenti self-report di valutazione dell'attaccamento nell'adulto con l'obiettivo di approfondire l'analisi dei costrutti e delle metodologie di indagine e di rispondere ai quesiti relativi alla relazione tra i diversi strumenti e alla loro convergenza o divergenza. Più di recente, riprendendo precedenti spunti di ricerca (ad esempio uno studio della corrispondenza fra categoria di attaccamento e il CCRT in coppie di amici) abbiamo utilizzato il CCRT come strumento capace di cogliere aspetti rilevanti della dinamica adolescente- genitore. In un lavoro del 2009 (Ortu, Guidi, \& Fantini, 2009) e volto a studiare il funzionamento sociale in adolescenza, abbiamo ad esempio utilizzato il CCRT per studiare la relazione tra schemi schemi relazionali disadattivi dell'adolescente (definiti nei termini di in termini di rigidità/flessibilità così come riflessa dalla pervasività del CCRT) e quelli del genitore. Il CCRT, ottenuto sulla base dell'intervista RAP (Relationship Anecdotes Paradigms; Luborsky, 1978) - una intervista appositamente costruita per sollecitare la narrazione di episodi relazionali - somministrata a 35 adolescenti e alle loro madri, è stato utilizzato per porre in luce l'influenza dei modelli relazionali genitoriali sui modelli emergenti in adolescenza.

Il confronto dei modelli relazionali tra i due gruppi ha messo in luce 
che il carattere disadattivo dello schema relazionale del genitore influenza il funzionamento attuale dell'adolescente. Alla luce di queste considerazioni preliminari di carattere metodologico e utilizzando la teoria dell'Attaccamento come sfondo, abbiamo iniziato, anche in collaborazione con altri gruppi di ricerca, una serie di studi per la valutazione del rischio psicopatologico e per i processi adattativi nelle diverse fasi del ciclo di vita.

Sulla base dei dati di ricerca che indicano nell'insicurezza dell'attaccamento un fattore di rischio per lo sviluppo di diverse condizioni psicopatologiche, abbiamo rivolto così la nostra attenzione alle problematiche sollevate dall'applicazione degli strumenti di valutazione dell'attaccamento al contesto clinico dell'adolescenza. In buona parte, tali problemi riguardano la relativa inadeguatezza di tali strumenti di valutazione nel cogliere alcune caratteristiche essenziali dello sviluppo affettivo e sociale dell'adolescente. A tale inadeguatezza si somma poi l'esigenza clinica di calibrare l'intervento secondo le caratteristiche di questa fase evolutiva.

Rispetto all'adolescenza, gli studi empirici hanno inoltre evidenziato una sostanziale difficoltà a rintracciare quella continuità temporale dei modelli di attaccamento che è alla base della prospettiva evolutiva per primo delineata da John Bowlby. In diversi studi è stato messo in luce che, se si tiene conto della classificazione tradizionale dei modelli di attaccamento (e cioè alla classificazione ottenute in base alla Strange Situation e all'Adult Attachment Interview, AAI) le valutazioni complessive di campioni di adolescenti presentano diverse peculiarità. Le classificazioni degli adolescenti si presentano cioè scarsamente correlate tanto alle classificazioni AAI degli adulti quanto a quelle dei bambini di 12-18 mesi alla Strange Situation: negli adolescenti la percentuale dei sicuri tende a diminuire in favore dei distanzianti che in questa fase sembrano essere sovrarappresentati (Main, 1991; Ammaniti, van Ijzendorn, Speranza, \& Tambelli, 2000; Sroufe, 2005). 
D'altro canto, gli studi longitudinali che valutano la stabilità dall'infanzia all'età adulta delle classificazioni dell'attaccamento dei singoli soggetti mettono in luce che classificazioni ottenute alla Strange Situation a 12 o 18 mesi consentono, rispetto alle valutazioni effettuate in adolescenza con l'AICA (Attachment Interview in Child and Adolescent; Ammaniti, Candelori, \& De Coro, 1990; Ammaniti et al. 2000) un adattamento dell'AAI per l'età adolescenziale, una migliore previsione della classificazione all'AAI in età adulta, configurando un vero e proprio gap nella valutazione dell'attaccamento in questa fascia di età ed evidenziando un quadro evolutivo di continuità a singhiozzo fra le diverse fasi del ciclo di vita.

Nel tentativo di rispondere agli interrogativi sollevati da questi dati, abbiamo tenuto conto del fatto che gli studi longitudinali evidenziano come il potere predittivo delle valutazioni effettuate nel corso dello sviluppo aumenti (e questo vale anche per l'adolescenza) se la valutazione dell'attaccamento in ciascun periodo evolutivo tiene conto non solo delle capacità acquisite, ma anche di quelle in via di acquisizione (dell'effetto delle esperienze precedenti sulle relazioni attuali ma anche dell'effetto delle nuove esperienze su quelle future). In adolescenza inoltre la predittività (e quindi la stabilità) delle valutazioni della qualità dell'attaccamento aumenta se vengono impiegate misure di tipo misto, ovvero, sia di tipo rappresentazionale che comportamentale (Sroufe, 2005).

La letteratura più recente, in particolare, sottolinea l'importanza della dimensione interattiva soprattutto nel rapporto genitore-adolescente.

Prendendo spunto dalle precedenti problematiche evidenziate dalla ricerca empirica sull'attaccamento in adolescenza e dalle necessità di una possibile applicazione al contesto clinico dell'adolescenza, ci siamo così posti due obiettivi essenziali:

- trovare un sistema di valutazione dell'attaccamento che sia omogeneo al costrutto elaborato da John Bowlby e Mary Ainsworth e, al tempo 
stesso, sia in grado di cogliere le evoluzioni nei domini cognitivo, motivazionale e sociale tipiche dell'adolescenza;

- verificare che questo sistema sia in grado di evidenziare le specifiche relazioni tra sviluppo dell'attaccamento, psicopatologia e organizzazione della personalità in questa fase del ciclo di vita.

Una prima fase del lavoro è dunque consistita nella identificazione degli strumenti di valutazione. Tenendo conto che uno strumento di valutazione ottimale deve:

- essere capace di cogliere il compito evolutivo e funzionamento specifico della fase - e cioè nel caso dell'adolescente gli aspetti essenziali del processo di organizzazione di una nuova rappresentazione di "Sé come agente" e di regolazione autonoma rispetto al proprio pensiero (ricerca dell'autonomia) all'interno di uno scambio interattivo con $\mathrm{il} / \mathrm{i}$ genitore/i;

- essere sensibile ai contesti d'interazione - e cioè capace di offrire una valutazione complessiva dell'attaccamento aderente a ciò che l'adolescente effettivamente fa nei diversi contesti, in particolare di quello familiare, e di come adolescente e genitore apprendono a regolare mutuamente i propri stati affettivi e le proprie prospettive mentali;

- permettere l'integrazione di misure osservative e rappresentazionali;

- cogliere in particolare l'interazione con altri sistemi motivazionali interpersonali;

- evidenziare aspetti rilevanti per la psicopatologia (attaccamento disorganizzato).

In vista di una valutazione degli schemi relazionali disadattativi abbiamo individuato nel sistema PIGA (Profilo d'Interazione GenitoreAdolescente; Lyons-Ruth, Hennigshausen, \& Holmes, 2003) uno strumento capace di soddisfare queste diverse esigenze. Il sistema PIGA - sviluppato nel corso di un importante studio longitudinale multicentrico condotto su un gruppo di bambini valutati in momenti 
diversi dello sviluppo, dalla prima infanzia all'età giovane adulta costituisce una griglia di osservazione specifica per studiare l'evoluzione dei comportamenti disorganizzati nel corso dello sviluppo. In particolare, esso costituisce il primo approccio metodologico allo studio delle strategie di tipo controlling che costituiscono l'evoluzione tipica dell'attaccamento disorganizzato nel corso dello sviluppo (Lyons-Ruth $\&$ Jacobvitz 1999). Rispetto agli studi che tipicamente si rivolgono all'Adult Attachment Interview (AAI) o al suo adattamento per l'adolescenza (AICA; Ammaniti, Candelori et al., 1990; Ammaniti, van Ijzendoorn et al., 2000), il sistema PIGA fa riferimento non solo a misure rappresentazionali dell'attaccamento, ma anche a osservazioni comportamentali di tipo interattivo. Questo sistema prevede una valutazione complessa che si fonda sull'analisi della videoregistrazione dell'interazione genitore-adolescente e porta a classificare le strategie interattive secondo dimensioni coerenti con quelle definite dalla Strange Situation e dell'Adult Attachment Interview. Questo sistema di valutazione ci è parso cioè capace di cogliere, alla luce dei compiti evolutivi dello funzionamento specifico della fase adolescenziale, l'equilibrio tra attaccamento ed esplorazione. I dati emersi da un lavoro preliminare di tipo metodologico, che aveva l'obiettivo di esplorare la validità del sistema PIGA, ci hanno permesso di sostenere che questo sistema offre: 1) una visione più integrata e adeguata alla fase dello sviluppo dell'attaccamento in adolescenza rispetto alla sola misura rappresentazionale fornita dall'applicazione dell'AICA (equivalente dell'AAI per l'adolescente); 2) una visione più accurata rispetto alla riorganizzazione dei sistemi motivazionali tipica dell'adolescenza; 3) la possibilità di cogliere in modo clinicamente accurato e significativo le modalità disadadattive dell'adolescente, con particolare riferimento all'evoluzione in questa fase delle strategie controlling tipiche dell'attaccamento disoganizzato.

I dati confortanti di questo primo lavoro ci hanno incoraggiato a 
proseguire in questa linea di ricerca. In un successivo lavoro (Manaresi, Williams, \& Cotugno, 2008), volto a esplorare il rapporto tra attaccamento e psicopatologia in adolescenza, svolto in collaborazione con i colleghi Manaresi e Cotugno del centro clinico ASTREA di Roma, abbiamo così affiancato al sistema PIGA un ulteriore strumento, l'AIMIT (Analisi degli Indicatori delle Motivazioni Interpersonali nei Trascritti) messo a punto da Liotti e collaboratori (Liotti \& Monticelli, 2008; Gruppo per lo studio delle motivazioni interpersonali in psicoterapia, 2008) che mira a fornire una analisi degli indicatori delle motivazioni interpersonali nei trascritti - per mettere in luce le motivazioni interpersonali sottese agli scambi interattivi e comunicativi genitoreadolescente. In questo recente lavoro (Manaresi, Williams, \& Cotugno, 2008) abbiamo dunque utilizzato il sistema PIGA e il sistema AIMIT per lo studio delle interazioni genitore-adolescente in due campioni di adolescenti, il primo costituito 16 diadi adolescente-genitore provenienti da una popolazione a basso rischio psicosociale e il secondo costituito da 27 diadi provenienti da una popolazione clinica di pazienti diagnosticati con disturbi di personalità. Utilizzando questi due strumenti, abbiamo valutato una situazione interattiva semistrutturata, in cui adolescente e genitore vengono chiamati a confrontarsi su specifiche tematiche-stimolo offerte da un operatore non coinvolto nel processo terapeutico. La procedura utilizzata è costituita da 6 episodi, della durata di 5 minuti ciascuno, introdotti da "indicazioni di discussione" che costituiscono stimoli stressanti di intensità crescente. Le interazioni così stimolate, vengono audio-videoregistrate e successivamente trascritte verbatim. Dopo la prima sequenza di discussione libera non focalizzata, il genitore viene sottoposto all'Adult Attachment Interview, mentre all'adolescente viene somministrata l'AICA. Le due interviste vengono audioregistrate e valutate utilizzando il sistema di codifica di Main e Goldwyn (1998).

In analogia con la metodologia che informa la Strange Situation, la 
nostra procedura osservativa è costituita da una sequenza prestabilita di situazioni indotte da stimoli fase-specifici, in grado di attivare il sistema di attaccamento-accudimento nell'interazione genitore-figlio.

Una volta effettuata la videoregistrazione dell'interazione semistrutturata, i dialoghi sono stati trascritti e analizzati secondo il metodo AIMIT. Le videoregistrazioni sono state valutate secondo le dimensioni PIGA sopra descritte. Le due valutazioni sono state effettuate in cieco da codificatori esperti dei due sistemi. I dati così raccolti hanno costruito un quadro sovrapponibile a quello ottenuto da Lyons-Ruth e collaboratori (2003), e cioè anche nella popolazione italiana studiata la qualità delle interazioni fra genitore e adolescente impegnati nella soluzione del compito decisionale mostra una correlazione positiva con la categoria di attaccamento del genitore e al tempo stesso si rivela più capace della categoria di attaccamento del genitore di predire la categoria di attaccamento dell'adolescente (Williams, Ardito, Ortu, \& Dazzi 2008). Questo dato è stato inoltre confermato da un altro studio che evidenziava la forte predittività dell'attaccamento rispetto alla modalità di gestione dell'aggressività fra genitore e adolescente, modalità che si presenta come funzionale nelle diadi con attaccamento sicuro e disfunzionali, con una marcata tendenza al passaggio all'atto - nella forma di vere e proprie aggressioni (fisiche o verbali) - nelle diadi insicure o disorganizzate (Williams, 2007; Williams, Aloi et al., 2008; Williams, Ardito et al., 2008).

Questa seconda modalità si presenta come fortemente ricorrente nel gruppo costituito da adolescenti con disturbi gravi di personalità (Williams, 2007; Williams, Aloi, Di Chio, Ortu, \& Lingiardi, 2008; Manaresi, Williams, \& Cotugno, 2008). L'analisi delle interazioni PIGA di questi giovani pazienti ha consentito di evidenziare la prevalenza delle interazioni traumatiche descritte dalla Lyons-Ruth attraverso le tipologie dei profili disorganizzati del sistema PIGA e ci ha condotti a ipotizzare come la disorganizzazione dell'attaccamento in adolescenza 
sia riconducibile alla impossibilità, in particolare per un adolescente segnato da una storia di traumi infantili (come gli adolescenti del nostro campione clinico) di conciliare le spinte all'acquisizione della nuova identità adolescenziale, di nuovi legami e nuove modalità di rapporto con i bisogni di attaccamento. Nel caso dell'attaccamento disorganizzato l'adolescente sembra sperimentare il percorso di differenziazione psicologica, che costituisce uno dei compiti evolutivi di questa fase evolutiva, come un attacco all'altro o come una rottura insanabile del legame. La plausibilità di questo tipo di interpretazione sembra essere confermata dal confronto tra i dati ottenuti dal sistema PIGA e dalla valutazione dei sistemi motivazionali fornita dal sistema AIMIT. Da tale confronto emerge con chiarezza che tanto la qualità dell'attaccamento del genitore, valutata mediante l'AAI, quanto la valutazione dell'interazione adolescente-genitore, effettuata attraverso il PIGA, sono fortemente predittive di strategie di tipo controlling caratterizzate dall'attivazione pervasiva e disarmonica del sistema motivazionale di rango in luogo di quello dell'attaccamento e dell'accudimento.

I futuri sviluppi di questa prospettiva di ricerca saranno rivolti ad approfondire maggiormente lo studio: a) della congruenza tra le valutazioni dell'attaccamento in adolescenza effettuate attraverso il sistema PIGA con lo sviluppo delle relazioni oggettuali cosi come analizzato nella prospettiva empirica messa a punto da Drew Westen con il metodo SCORS (1991b, 1996) e b) della capacità del sistema PIGA e del sistema AIMIT di cogliere le differenze cliniche più rilevanti tra gruppi di disturbi di personalità del cluster A e del cluster B.

\section{Conclusioni}

Il lavoro del gruppo di ricerca ha seguito delle linee distinte che si rivolgono a due aspetti di stretta rilevanza per lo studio empirico della psicoterapia: la comprensione dei diversi fattori che concorrono 
all'evoluzione del processo nella prospettiva psicodinamica; l'identificazione delle caratteristiche dell'organizzazione dei processi dell'attaccamento che presentano maggiore salienza per lo sviluppo della patologia di personalità e degli schemi relazionali disadattativi nel contesto della psicoterapia.

Per quanto concerne gli studi sul processo, le nostre ricerche hanno consentito di evidenziare la complessità delle interazioni tra i diversi fattori che favoriscono od ostacolano l'evoluzione del processo terapeutico. In particolare, è stato possibile evidenziare che la valutazione accurata del cambiamento clinico non può essere realizzata attraverso un unico costrutto clinico. Le diverse dimensioni prese in esame dai diversi strumenti di valutazione del cambiamento presentano solo un grado relativo di omogeneità. Così, ad esempio, l'evoluzione degli schemi relazionali disadattativi non va di pari passo con l'evoluzione delle organizzazioni dei meccanismi di difesa nell'ambito del processo terapeutico. Se si raffrontano le evoluzioni di queste singole dimensioni cliniche nel corso di una terapia con altri indicatori dell'efficacia del processo (ad es., l'alleanza terapeutica) o con l'outcome della terapia stessa si perviene a conclusioni parziali e talora errate. Più proficuo sembra un approccio di valutazione multi-dimensionale che consenta di valutare l'andamento del processo attraverso le specifiche interazioni che $i$ singoli fattori mostrano nelle diverse fasi del trattamento.

Per quanto concerne lo studio delle valutazioni dell'attaccamento, i risultati ottenuti consentono una prima ridefinizione delle classificazioni tradizionali dell'attaccamento alla luce di alcune dimensioni di particolare rilievo per la psicoterapia. Con riferimento all'ambito della patologia adolescenziale, è stato in particolare possibile costruire uno strumento di valutazione che sia ancorato al costrutto originario di attaccamento e che mostri, al tempo stesso, l'intreccio tra l'evoluzione di questo sistema con le vicissitudini evolutive e motivazionali tipiche di 
164

Ricerca in Psicoterapia / Research in Psychotherapy 2010; 2(13): 147-167 http://www.researchinpsychotherapy.net

questa fase. In particolare, è stato possibile specificare quali aspetti del modello disorganizzato dell'attaccamento si presentano in modo ricorrente e distintivo nelle organizzazioni patologiche della personalità. Come evidenziato dall'applicazione di questo approccio ad alcuni casi clinici, tale impostazione suggerisce una linea interpretativa che a partire dalle valutazioni dell'attaccamento consente di formulare delle previsioni circa le modalità e i contenuti attraverso cui gli schemi disadattativi di origine traumatica si manifestano nel contesto clinico.

\section{Bibliografia}

American Psychiatric Association (2000). Manuale diagnostico e statistico dei disturbi mentali - Text revision (DSM-IV-TR). Tr. it. Masson, Milano 2002.

Ammaniti, M., Candelori, C., \& De Coro, A. (1990). IAL: Intervista sull'attaccamento nella Latenza (versione A.I.C.A: Attachment Interview for Childhood and Adolescence). Manoscritto non pubblicato, Dipartimento di Psicologia Dinamica e Clinica, "La Sapienza", Università degli Studi di Roma.

Ammaniti, M., van Ijzendoorn, M.H., Speranza, A.M., \& Tambelli, R., (2000). Internal Working Models of attachment during late childhood and adolescence: an exploration of stability and change. Attachment and Human Development, 2(3), 328-346.

Barone, L., \& Del Corno, F. (2007). La Valutazione dell'Attaccamento Adulto. Milano: Raffaello Cortina.

Bucci, W. (1997a). Patterns of discourse in "good" and troubled hours. A multiple code interpretation. Journal of the American Psychoanalytic Association, 45, 155-187.

Bucci, W. (1997b). Psicoanalisi e scienza cognitiva. Roma: Fioriti Editore.

Colli, A., \& Lingiardi, V. (2001, Ottobre). Una proposta di valutazione dell'alleanza terapeutica a partire dai trascritti delle sedute: il sistema IVAT. Relazione presentata al Convegno Nazionale SPR-Italia, Palermo.

Colli, A., \& Lingiardi, V. (2002). Indice di valutazione dell'alleanza terapeutica (IVAT). Una proposta di valutazione dell'alleanza terapeutica a partire dai trascritti delle sedute. In V. Lingiardi (Ed.), L'alleanza terapeutica. Teoria, clinica, ricerca (pp. 211-222). Milano: Raffaello Cortina

Colli, A., \& Lingiardi, V. (2009). The Collaborative Interactions Scales: a new transcribed-based method for the assessment of the therapeutic alliance ruptures and resolutions in psychotherapy. Psychotherapy Research, 19(6), 718-734.

Dazzi, N., De Coro, A., Ortu, F., Andreassi, S., Cundari, M., Ostuni, V., Petruccelli, I., \& Sergi, G. (1998b). Il CCRT in un campione italiano di psicoterapie: uno studio della relazione fra categorie su misura e categorie standard. Ricerca in Psicoterapia, 1(2), 205-223. 
De Coro, A., Andreassi, S., \& Dazzi, N. (2001, Settembre). Lo studio dei modelli cognitivo-affettivi delle relazioni. Un adattamento del CCRT per la diagnosi psicodinamica. Relazione presentata al Congresso Nazionale della sezione di Psicologia Clinica della Associazione Italiana di Psicologia, Mondello.

De Coro, A., Ortu, F., Speranza, A.M., Andreassi, S., \& Pazzagli, C. (2003). Referential Activity and Conversational Incoherence in Attachment Interview applied to young Adolescents. Paper presented at the European Congress of SPR - Weimar (Germany).

Fonagy, P., \& Target, M. (1997). Attaccamento e Funzione Riflessiva. Tr. it. Raffello Cortina, Milano 2001.

Fonagy, P., Steeele, M., Steele, H., \& Target, M. (1998). Reflective-Functioning Manual (Version 5). Unpublished manuscript, University College London, London.

Gruppo per lo studio delle motivazioni interpersonali in psicoterapia (2008). Il manuale AIMT. In G. Liotti \& F. Monticelli (Eds.), I sistemi motivazionali nel dialogo clinico. Il manuale AIMIT (pp. 157-214). Milano: Raffaello Cortina.

Lichtenberg, J.D. (1989). Psychoanalysis and Motivation. Hillsdale-London: The Analytic Press (tr. it. Psicoanalisi e sistemi motivazionali, Raffaello Cortina, Milano 1995).

Lichtenberg, J.D., Lachmann, F.M., \& Fosshage, J.L. (1996). The clinical exchange. The Analytic Press, Inc. (tr. it. Lo scambio clinico, Raffaello Cortina, Milano, 2000).

Lingiardi, V., \& Madeddu, F. (2002). I meccanismi di difesa. Teoria, valutazione, clinica. Milano: Raffaello Cortina.

Liotti, G., \& Monticelli F. (2008). I sistemi motivazionali nel dialogo clinico. Il manuale AIMIT. Milano: Raffaello Cortina.

Luborsky, L. (1978). The self-interpretation of the relationship anecdotes paradigms (RAP) interview. Unpublished manuscript, Department of Psychiatry, University of Pennsylvania.

Luborsky, L., \& Crits-Christoph, P. (1990). Understanding Transference: The CCRT Method. New York: Basic Books (tr. it. Capire il transfert, Raffaello Cortina, Milano 1992).

Lyons-Ruth, K., \& Jacobvitz, D. (1999). Attachment Disorganization: Unresolved Loss, Relational Violence and Lapses in Behavioral and Attentional Strategies. In J. Cassidy \& P. Shaver (Eds.), Handbook of Attachment (520-554). New York, London: Guilfrod Press.

Lyons-Ruth, K., Hennighausen, K., \& Holmes, B. (2003). Parent-Adolescent Coding Manual. Unpublished Manuscript, Department of Psychiatry, Cambridge (MA).

Main, M. (1991). Metacognitive knowledge, metacognitive monitoring and singular (coherent) vs. multiple (incoherent) model of attachment. In Parkes, C.M., Stevenson-Hinde J. \& Marris, P. (Eds.), Attachment across the life cicle. London: Routledge. (Tr. it. Conoscenza metacognitiva, monitoraggio metacognitivo e modello di attaccamento unitario (coerente) vs modello di attaccamento multiplo (incoerente). In C.M. Parkes, J. StevensonHinde \& P. Marris (Eds.), L'attaccamento nel ciclo di vita. Il Pensiero Scientifico, Roma 1995).

Main, M., \& Goldwyn, R. (1998). Adult Attachment Scoring and Classification Systems. Unpublished manuscript, University of California, Berkeley.

Manaresi, F., Williams, R., \& Cotugno, A. (2008). Sistemi motivazionali interpersonali in adolescenza. Psicobiettivo, 1, 32-47. 
Ortu, F., Cascioli, A., Pazzagli, C., Piscicelli, S., Williams, R., \& Dazzi, N., (2001, Settembre). Uno studio metodologico del CCRT di Luborsky: la relazione fra la struttura narrativa deglui episodi relazionali $e$ l'organizzazione della personalità. Relazione presentata al III Congresso Nazionale della sezione di Psicologia Clinica della Associazione Italiana di Psicologia, Mondello.

Ortu, F., Lingiardi, V., Pazzagli, C., Williams, R., \& Dazzi, N. (2001, March). Application of CCCRT, RA, DMRS to evaluate the Psychotherapy Process. A comparison between successful and dropped-out cases. Paper presented at the European Congress of the Society for Psychotherapy Research, Leiden, Holland.

Ortu, F., Williams, R., \& Dazzi, N. (2004, June). Una proposta metodologica di estensione del CCRT di Luborsky. Paper presented at the 35th SPR International Meeting, Rome, Italy.

Ortu, F., Guidi, A., \& Fantini, F. (2009, Settembre). Un applicazione del CCRT alle diadi madre-adolescente in un campione romano. Relazione presentata al XV Convegno della sezione clinica dell'AIP, Chieti.

Perry, J.C. (1990). Defense Mechanism Rating Scale (5th edition). Unpublished manual. Cambridge University, Boston.

Perry, J.C. (1990). Defense Mechanism Rating Scale. Tr. it. in V. Lingiardi \& F. Madeddu (Eds.), I meccanismi di difesa (nuova ed.) (pp. 381-458). Raffaello Cortina, Milano 2002.

Sroufe, A. (2005). The development of the person. New York: Wiley.

Steele, H., \& Steele, M. (2010). Adult Attachment Interview - Applicazioni cliniche. Milano: Raffaello Cortina.

Westen, D. (1991b). Social cognition and object relations. Psychological Bullettin, 109, 3, 429-455.

Westen, D. (1996). Social cognition and object relations scales for interview in narrative data. Vedi sito internet http://www.psychsystem.net/lab.

Westen, D., Shedler, J., \& Lingiardi, V. (2003). La valutazione della personalità con la SWAP-200. Milano: Raffello Cortina.

Williams, R. (2007). Qualità dell'attaccamento e patologia di personalità in adolescenza: uno studio empirico in un campione di pazienti romani. Relazione presentata alla Giornata Nazionale dei Contributi dei Giovani Ricercatori di Psicologia Evolutiva, Clinica-Dinamica, Sociale. Atti del convegno, pp. 54-60.

Williams, R., Aloi, A., Di Chio, A., Ortu, F., \& Lingiardi, V. (2008, Settembre). La valutazione per prototipi con la SWAP-200-A: un'applicazione a un campione romano di adolescenti. Relazione presentata al VII Congresso della Sezione Italiana della SPR, Modena.

Williams, R., Ardito, D., Ortu, F., \& Dazzi, N. (2008, Gennaio). La valutazione dell'attaccamento e dell'intersoggettività in adolescenza. Relazione presentata al $9^{\circ}$ Convegno Nazionale dei Gruppi Italiani di Psicoterapia Psicoanalitica dell'Adolescenza AGIPPsA "Le Nuove frontiere della ricerca clinica in adolescenza", Roma.

Williams, R., Ferrara, M., Aloi, A., \& Gazzillo, F. (2009). La valutazione della patologia di personalità con la SWAP-200-A: un'applicazione ad un campione clinico di adolescenti italiani. Infanzia e Adolescenza, 8(2), 33-34.

Williams, R., Ferrara, M., Ortu, F., Caratelli, A., \& Dazzi, N. (2005, Settembre). Uno studio sulla patologia di personalità in adolescenza. Relazione 
presentata al VI Convegno Nazionale della Sezione di Psicologia Clinica dell'AIP, San Benedetto del Tronto.

Williams, R., Ortu, F., Lingiardi, \& Dazzi, N. (2004, June). L'alleanza terapeutica e il continuuum espressivo-supportivo: uno studio empirico del processo in psicoterapia psicodinamica. Paper presented at the $35^{\text {th }}$ SPR International Meeting, Rome, Italy.

Williams, R., Saracino, D., Traverso, L., Ortu, F., \& Dazzi, N. (2005, March). Fluctuations in the therapeutic alliance with respect to the therapist's interventions in the treatment of a borderline patient. Paper presented at the $2^{\text {nd }}$ Joint Meeting of the SPR European and UK Chapters, Lausanne, Switzerland.

\begin{abstract}
Our research group, currently operating at the Department of Dynamic and Clinic Psychology, have mainly focused on two topics: the study on therapeutic process (in the perspectives of both empirical and conceptual research); the relevance of attachment theory for the understanding of abnormal personality development and its manifestations within the clinical context, with specific reference to adolescence.
\end{abstract}

\title{
Key words
}

Process, CCRT, adolescence, attachment theory, motivational systems 